\title{
EFECTOS DE LA CEPA NATIVA DE Trichoderma sp. Y LIXIVIADO DE VERMICOMPOST BOVINO SOBRE EL CRECIMIENTO FOLIAR Y CONTENIDO DE CLOROFILA EN ARROZ (Oryza sativa L.) EN FASE DE SEMILLERO
}

\author{
Chávez Vergara, Jorge Antonio ${ }^{a c^{*}}$; Torres García, Caridad Antonio ${ }^{\mathrm{b}}$; Espinoza Vera, Edixon Agustín'; \\ Zambrano Pazmiño, Diego Efrén ${ }^{\text {d; }}$ Villafuerte Barreto, Ariel Gonzalob; Zambrano Gavilanes, \\ Freddy Eli ${ }^{\mathrm{b}}$; Velázquez Vinces, José Ademar \\ a Universidad Técnica de Manabí, Facultad de Ingeniería Agronómica, \\ Campus La Teodomira, Lodana, Ecuador. \\ ${ }^{\mathrm{b}}$ Ministerio de Agricultura y Ganadería, Av. Olmedo y Sucre Edif. La Previsora, Portoviejo, Ecuador. \\ c Escuela Superior Politécnica Agropecuaria de Manabí, Manuel Félix López, 10 de Agosto 82 y Granda \\ Centeno, Calceta, Ecuador.

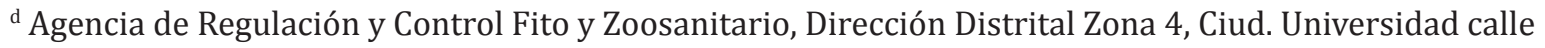 \\ U10 Av. 2, Edif. Burgos, Manta, Ecuador
}

Ingresado: 07/01/2020

\section{Resumen}

El arroz es uno de los granos más importantes en el Ecuador en términos de valor económico y consumo. Este trabajo tuvo como objetivo evaluar el efecto de la aplicación de una cepa nativa de Trichoderma sp. y el lixiviado de vermicompost de estiércol bovino (LVCB) sobre el cultivo de arroz (Oryza sativa L), variedad SFL-11 en fase de semillero. Se emplearon ocho tratamientos (seis con Trichoderma sp., uno con lixiviado de vermicompost de estiércol bovino y el testigo agua) en cuatro replicas, distribuidos en bloques completamente al azar (DBCA). La investigación se realizó en la parroquia Charapotó del cantón Sucre de la provincia de Manabí. A los 25 días después de la siembra se evaluaron el número de hojas, masa seca foliar y pigmentos clorofílicos. Con los datos obtenidos de las variables respuesta, se aplicó un análisis de varianza y las medias fueron comparadas mediante la prueba de Duncan con el nivel de significancia al 5\%. La aplicación de la cepa nativa de Trichoderma sp. incrementó el número de hojas, masa seca foliar y pigmentos clorofílicos en las plántulas de arroz variedad SFL-11. La imbibición de la semilla de arroz variedad SFL-

\footnotetext{
* Universidad Técnica de Manabí, Programa de Maestría en Agronomía Mención Producción Agrícola Sostenible, Av. Urbina y Che Guevara, Portoviejo. Ministerio de Agricultura y Ganadería, Av. Olmedo y Sucre Edif. La Previsora, Portoviejo, Ecuador. Teléfono: +593996002390 . Correo electrónico: jchfm@hotmail.com
}

Aceptado: 08/06/2020

11 en el bioestimulante líquido de vermicompost de estiércol bovino en dilución 1:10 indujeron incrementos significativos en masa seca foliar, numero de hojas y el contenido de clorofilas en las plántulas de arroz variedad SFL-11.

Palabras clave: Bioestimulantes, biomasa foliar, estiércol de bovino, pigmentos clorofílicos, Trichoderma.

FECTS OF NATIVA STRAIN OF Trichoderma sp. AND BOVINE VERMICOMPOST LEACHATE ON FOLIAR GROWTH AND CHLOROPHYLL CONTENT OF RICE (Oryza sativa L.) IN SEEDLING PHASE

\begin{abstract}
Rice is one of the most important grains in Ecuador in terms of economic value and consumption. The objective of this work was to evaluate the effect of the application of a native strain of Trichoderma sp. and the vermicompost leachate from bovine manure (LVCB) on the rice crop (Oryza sativa L.), variety SFL-11 in the seedbed phase. Eight treatments (six with Trichoderma sp., one with vermicompost leachate from bovine manure and the water control) were used in four replicates, distributed in completely random blocks (DBCA). The investigation was carried out
\end{abstract}


in the Charapoto parish of Sucre canton of Manabí province. At 25 days after sowing, the number of leaves, dry leaf mass and chlorophyll pigments were evaluated. With the data obtained from the response variables, an analysis of variance was applied, and the means were compared using the Duncan test with the significance level at 5\%. The application of the native strain of Trichoderma sp. increased the number of leaves, dry leaf mass and chlorophyll pigments in the seedling rice variety SFL-11. The imbibition of the rice seed variety SFL-11 in the liquid biostimulant of bovine manure vermicompost in 1:10 dilution induced significant increases in mass dry foliar, number of leaves and the content of chlorophylls in the seedling of rice variety SFL-11.

Keywords: Biostimulants, cattle manure, chlorophyll pigments, foliar biomass, Trichoderma.

\section{INTRODUCCIÓN}

El arroz (Oriza sativa L.) proporciona entre el 66\% al $70 \%$ de la ingesta de calorías corporales a los consumidores, constituyendo un rubro importante en la soberanía y seguridad alimentaria [1]. En el Ecuador, la superficie sembrada en el año 2017 fue de 370.406 hectáreas, con una producción de 1'440.865 T.M. siendo Guayas (70,11\%) y Los Ríos $(24,14 \%)$ las provincias de mayor producción en el país. Sin embargo, a pesar de que se cuenta con una cantidad considerable de hectáreas sembradas en el país, el rendimiento por hectárea es bajo [2]. Los fertilizantes químicos se consideran parte esencial de la agricultura convencional. Algunos estudios mencionan que su aplicación directa o indirecta puede provocar una serie de cambios en las propiedades físicas, químicas y biológicas del suelo $[3,4]$. Por el contrario, el estiércol orgánico es una fuente casi completa de la mayoría de los nutrientes y está disponible durante más tiempo debido a la descomposición lenta y la liberación gradual de los nutrientes [5]. El uso equilibrado de nutrientes a través de fuentes orgánicas como el estiércol, el vermicompost, el abono verde, la torta de neem y los biofertilizantes son requisitos previos para mantener la fertilidad del suelo y producir el máximo rendimiento de cualquier cultivo [6].

Por otro lado, las sustancias húmicas que son una colección de compuestos heterogéneos, originalmente categorizadas de acuerdo a su peso molecular y solubilidad [7], tienen acción de bioestimulante destacando la relación entre las propiedades químicas del material humificado [8]. Las sustancias húmicas del lixiviado de vermicompost proporcionan un nicho adecuado en el suelo para el desarrollo microbiano; además de tener un alto potencial para aumentar la producción vegetal por la cantidad en su composición de elementos minerales, hormonas vegetales y aminoácidos incluso en condiciones ambientales desfavorables [9].

La etapa inicial del crecimiento del arroz, cuando el follaje aún se encuentra joven y frágil, es donde existe mayor predisposición a diferentes daños de origen biótico y abiótico [10]. Uno de los principales problemas existentes en el cultivo del arroz es su escaso crecimiento en la etapa inicial (plántulas) cuando se encuentra en semillero. Una alternativa para estimular su crecimiento es el uso efectivo de Trichoderma sp., el cual ha sido probado en diferentes cultivos con excelentes resultados [11] en la etapa de semillero como promotor de crecimiento en plántulas [12] al liberar a nivel radicular auxinas [13] y sustancias relacionadas con el crecimiento vegetal como sesquiterpenoides, péptidos cíclicos [14] citoquininas y ácido indol acético (IAA) que actúan como catalizadores de tejidos meristemáticos primarios acelerando la reproducción celular lo cual se traduce en aumento de masa foliar [15] pero no se tiene establecida una frecuencia de su aplicación que estimule de manera eficiente el desarrollo vegetativo en el cultivo del arroz. Las bondades que presenta este hongo han posibilitado la elaboración de productos biológicos con características amigables con el ambiente, pero el éxito está amparado en la precisa selección de cepas; esto refuerza la necesidad de efectuar una correcta selección de los aislamientos respecto a su ambiente para obtener resultados consistentes en condiciones de campo [16].

La variedad de arroz SFL-11 es la de mayor superficie de siembra en la parroquia Charapotó, principal zona productora de arroz en la provincia de Manabí-Ecuador, debido a que sus características la hacen ideal para el agricultor que busca calidad y rendimiento en un cultivo bajo riego. Las características agronómicas son porcentaje de germinación mayor a 90\%, altura de planta de $126 \mathrm{~cm}$, macollamiento intermedio, ciclo de cultivo entre 127-131 días y rendimiento de 6-8 t ha ${ }^{-1}$; mientras que, las características de grano son índice de pilado $67 \%$, grano largo $(7,5$ $\mathrm{mm}$ descascarado), desgrane intermedio y carece de centro blanco [17]. 
Con base en lo anterior, el objetivo de la presente investigación fue evaluar los efectos de la aplicación de una cepa nativa de Trichoderma sp. y el lixiviado de vermicompost de estiércol bovino (LVCB) en el cultivo del arroz (Oryza sativa L.) variedad SFL-11 en fase de semillero, que es la etapa para obtener plantas con características morfológicas adecuadas para ser llevadas a condiciones de campo.

\section{METODOLOGÍA}

\section{Ubicación}

La investigación se realizó en Puerto Cañita, localizado en la parroquia Charapotó, del cantón Sucre, provincia de Manabí, coordenadas: 557029, 9904620 UTM, a una altitud de $3 \mathrm{~m}$ s.n.m. con una temperatura promedio anual de $25,5{ }^{\circ} \mathrm{C}$; precipitación media aproximada de 347 milímetros; suelos de textura franco-arcillosa, $\mathrm{pH} 7,30$, contenido de materia orgánica 3,26\%.

\section{Procedencia de la cepa nativa de Trichoderma sp.}

Se utilizó una cepa nativa aislada en suelo de la parroquia Membrillo, cantón Bolívar, provincia de Manabí; el suelo era franco (48\% de arena, $40 \%$ de limo y $12 \%$ de arcilla), con pH de 6,6. Para la reproducción del hongo, se pre-coció 200 gramos de arrocillo y se colocó en una funda de celulosa, la cual fue cerrada con cinta adhesiva para proceder a esterilizar mediante autoclavado a $121^{\circ} \mathrm{C}$ y 15 PSI durante 15 minutos. Una vez enfriado, se lo llevó a una cámara de flujo laminar para efectuar la inoculación, colocando el arrocillo en un envase de polipropileno y transfiriendo un cuadrado de $0,5 \times 0,5 \mathrm{~cm}$ del medio de cultivo con la cepa de Trichoderma sp. Después de este procedimiento, se selló el envase y se lo llevó a un cuarto de incubación durante 15 días para su reproducción.

Después de ese tiempo el material fue lavado con agua destilada y cernido para la extracción del hongo, seguidamente se hicieron diluciones seriadas hasta la concentración de esporas $1 \mathrm{x}$ $10^{-8} \mathrm{UFC} / \mathrm{mL}$ mediante conteo de esporas en cámara de Neubauer a través del microscopio. La preparación de la solución se hizo en el laboratorio de Biología de la Escuela Superior Politécnica Agropecuaria de Manabí-ESPAM.

\section{Procedencia del lixiviado de vermicompost bovino}

El lixiviado de vermicompost de estiércol bovino utilizado fue producto de la deyecciones de la lombriz roja californiana (Eisenia foetida) criadas en un lecho de vermicompostaje, mismo que era una estructura de madera a manera de cajón construida desde 1 metro de la superficie del suelo y con dimensión de 1 metro de ancho por 5 metros de largo y con un pequeño desnivel para recuperar los efluentes o lixiviados. Las lombrices se alimentaron con residuo orgánico, previamente fermentado por 15 días, proveniente del estiércol bovino; diariamente se humedeció con agua el sustrato del lecho y después de 5 semanas se empezó a recolectar el lixiviado de vermicompost de estiércol bovino utilizado en esta investigación. Estos lechos pertenecen a la Asociación Agrícola Paraíso de los Ceibos, ubicada en Cañita, parroquia Charapotó, cantón Sucre, provincia de Manabí.

La composición química de los lixiviados de vermicompost fueron: Nitrógeno $(1,52 \%)$, fósforo $(0,65 \%)$, potasio $(1,6 \%)$, calcio $(0,58 \%)$, magnesio $(0,12 \%)$ hierro (1606 ppm), ácidos húmicos (6,6\%), ácidos fúlvicos (4,3\%) y materia orgánica $(18,17 \%)$.

\section{Manejo Agronómico}

Se utilizó 20 kilogramos de semilla de arroz variedad SFL-11, la cual fue sometida a imbibición en agua durante 24 horas, luego puesta al ambiente por 48 horas, cubriéndola totalmente con lona para su pre-germinación. El área para el semillero se preparó mediante fangueo utilizando un motocultor; este fue nivelado para el adecuado manejo del agua, y la siembra de la semilla pregerminada se realizó manualmente al voleo. Para el control de insectos-plaga se hizo una aplicación con Profenofos en dosis de 1,25 cc/litro de agua y una fertilización nitrogenada con urea al 46\%, al voleo.

\section{Tratamientos y metodología de evaluaciones}

Los tratamientos fueron distribuidos en bloques completamente al azar en cuatro repeticiones, teniendo un total de 32 unidades experimentales de $2 \mathrm{~m}^{2}$ cada una. Se evaluaron ocho tratamientos, seis con Trichoderma sp. 
en las diferentes dosis detalladas en la Tabla 1, aplicados a los 5 y 15 días después de la siembra. El lixiviado únicamente se utilizó en el tratamiento 7 , que consistió en la imbibición de la semilla de arroz en 10 mililitros de lixiviado de vermicompost bovino por litro de agua durante 24 horas, después se puso a secar por 48 horas, cubriéndola totalmente con una lona para su pre-germinación y posterior siembra. El

Tabla 1. Descripción de tratamientos, dosis y tiempo de aplicación

\begin{tabular}{|c|c|c|}
\hline Tratamientos & Dosis & Tiempo \\
\hline 1 & $2 \mathrm{~mL}$ & 5 DDS \\
\hline 2 & $2 \mathrm{~mL}$ & $15 \mathrm{DDS}$ \\
\hline 3 & $4 \mathrm{~mL}$ & $5 \mathrm{DDS}$ \\
\hline 4 & $4 \mathrm{~mL}$ & $15 \mathrm{DDS}$ \\
\hline 5 & $6 \mathrm{~mL}$ & $5 \mathrm{DDS}$ \\
\hline 6 & $6 \mathrm{~mL}$ & $15 \mathrm{DDS}$ \\
\hline 7 & $10 \mathrm{~mL}$ & Pre germinación \\
\hline 8 & Agua & Testigo sin aplicación \\
\hline
\end{tabular}

DDS: días después de la siembra

Dosis 1: $2 \mathrm{~mL} 1 \times 10^{-8}(\mathrm{UFC} / \mathrm{mL})$

Dosis 2: $4 \mathrm{~mL} 1 \times 10^{-8}(\mathrm{UFC} / \mathrm{mL})$

Dosis 3: $6 \mathrm{~mL} 1 \times 10^{-8}$ (UFC/mL)

Tratamiento 7: 10 mililitros lixiviado de vermicompost bovino (LVCB)/litro de agua para imbibición de semilla de arroz por 24 horas en fase de pre germinación.

tratamiento 8 fue el testigo (Tabla 1 ).

Se evaluó la masa seca foliar de 5 plantas del área útil de cada tratamiento a los 25 DDS. Se obtuvo la parte aérea de cada planta, se colocaron en bolsas de papel, se llevaron a estufa a $80^{\circ} \mathrm{C}$ por 24 horas, y se registró el peso en miligramos. El número de hojas se determinó de 5 plantas del área útil de cada tratamiento, se contabilizó el total de hojas de cada planta a los 25 DDS. Los pigmentos clorofílicos se registraron sobre el tercio superior de la lámina foliar de la hoja con mayor altura y se determinaron en 10 hojas de diferentes plantas del área útil de cada tratamiento; el contenido de pigmentos clorofílicos se registró con el dispositivo portátil SPAD 502 (25 DDS), en unidades SPAD.

\section{Análisis estadístico}

Los datos obtenidos para cada una de las variables se sometieron a una prueba de análisis de varianza y una prueba de comparación de medias de Duncan $(\mathrm{p}<0,05)$, con el software SPSS de IBM v.21.

\section{RESULTADOS Y DISCUSIÓN}

\section{Masa seca foliar}

Para la variable masa seca foliar se encontraron diferencias estadísticamente significativas $(\mathrm{p}<0,05)$ entre los tratamientos analizados, como se observa en la Fig. 1. El tratamiento que indujo la mayor masa seca foliar fue el de la cepa nativa de Trichoderma sp. en la dosis de $4 \mathrm{~mL}$ aplicado a los 15 días después de la siembra, destacando que los tratamientos 4 mL-5 DDS y LVCB 1:10 alcanzaron resultados similares y se encuentran después del mejor tratamiento. Se puede demostrar que la aplicación de Trichoderma sp. logró incrementar la masa seca foliar entre $46 \%$ y $59 \%$ y el LVCB un $56 \%$ en comparación a la masa seca foliar de las plantas testigo sin aplicación.

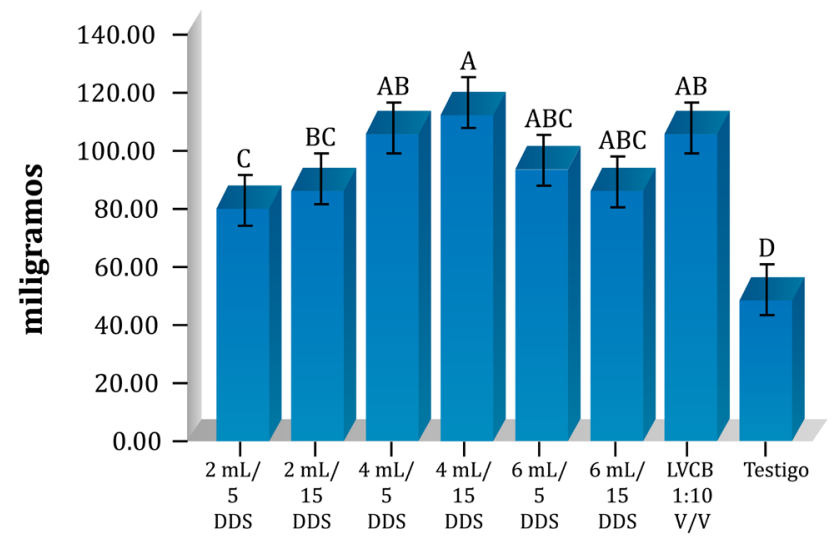

Fig. 1: Valores medios de masa seca foliar (mg) a las aplicaciones de Trichoderma sp. y lixiviados de vermicompost bovino (LVCB). Medias con una letra común no son significativamente diferentes para el test de Duncan ( $\mathrm{p}>0,05)$

La acumulación de masa seca sigue un patrón entre los órganos de las plantas, en el orden de masa seca foliar, masa seca de tallo y por último masa seca de raíces [18]. El resultado concuerda con Cubillos, Valero y Mejía [19], quienes reportaron incrementos en masa seca, al realizar aplicaciones en cultivo de maracuyá con cepa nativa de Trichoderma harzianum en dosis de $4 \mathrm{~mL}$ en concentración de $10^{-8} \mathrm{UFC}$; así mismo se reporta incrementos de masa seca de hojas en arveja después de la aplicación de Trichoderma sp. nativo a $1 \times 10^{-8}$ UFC [20]; aquello se atribuye a la capacidad del hongo para estimular el crecimiento vegetal en respuesta a la absorción de nutrientes, producción de auxinas, citoquininas y ácidos indol acético (IAA) que actúan como catalizadores de tejidos meristemáticos primarios acelerando la reproducción celular lo cual se traduce en un rápido y mayor desarrollo vegetativo [21,22]. 
El lixiviado de vermicompost bovino en imbibición de semillas coincide con el aumento de masa seca foliar, similar a lo reportado en una evaluación del efecto de los ácidos húmicos sobre los parámetros de calidad en plántulas de tomate [23], debido al efecto directo en el transporte iónico, favoreciendo la absorción de nutrientes, hormonas vegetales, aumentando velocidad sintética de ácidos nucleicos, mejorando la síntesis y velocidad de reacciones enzimaticas del ciclo de Krebs, obteniendo como resultado mayor producción de ATP, la cual es utilizada para diversas reacciones metabólicas [24]. Además, los productos orgánicos derivados del líquido de lombricomposta como tratamiento de semillas tienen efecto en uniformizar el desarrollo inicial de plántulas en arroz [25].

\section{Número de hojas}

Con respecto al número de hojas, se observan diferencias estadísticamente significativas ( $\mathrm{p}<0,05)$ (Fig. 2). El mayor número de hojas lo indujo LVCB-1:10, mientras que Trichoderma sp. $6 \mathrm{~mL}$ aplicado a los 5 y 15 DDS demostraron similitud en los resultados y se ubican después del mejor tratamiento. La imbibición de la semilla de arroz en LVCB incrementó el número de hojas un 26\%, mientras que las dosis de Trichoderma sp. aumentó entre 9\% y 22\% en comparación al número de hojas de plantas sin aplicación.

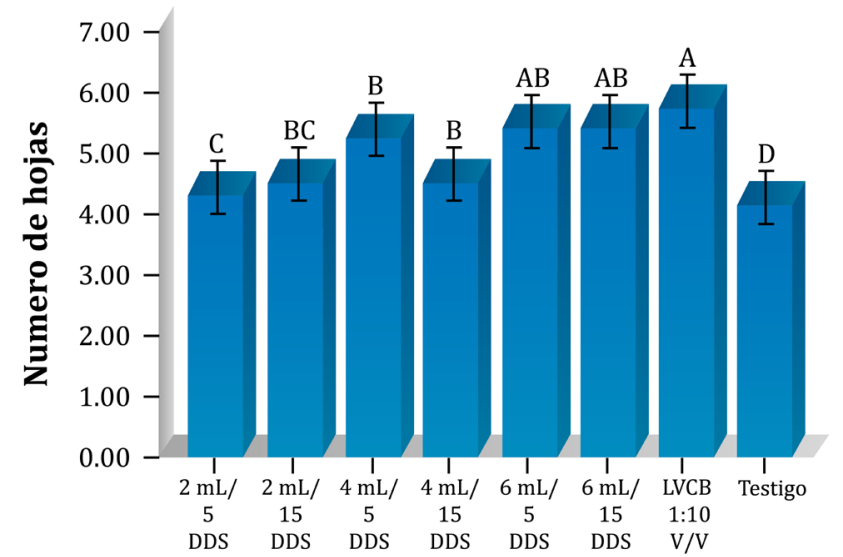

Fig. 2: Valores medios de número de hojas a las aplicaciones de Trichoderma sp., y lixiviados de vermicompost bovino (LVCB). Medias con una letra común no son significativamente diferentes para el test de Duncan ( $p>0,05)$

Los resultados concuerdan con la investigación realizada por Rodríguez, Pérez y Rodríguez [26] en tomate cuyos resultados presentados indican la influencia positiva al incremento de número de hojas después de imbibición de semilla en sustancias húmicas extraídas del vermicompost. Si bien es cierto son escasos los estudios que demuestran los efectos bioestimulantes de imbibición de semillas de arroz en lixiviados de vermicompost bovino, se sugiere que la acción bioestimulante de las sustancias húmicas se caracterizan por expresar desarrollo en raíces y brotes relacionados con la absorción, asimilación y distribución de nutrientes [27]. El resultado del tratamiento con valor mayor de número de hojas es atribuible al contenido de elementos nutritivos como nitrógeno, fósforo, potasio, calcio, magnesio, hierro y extractos húmicos como ácidos húmicos y fúlvicos presentes en el LVCB y determinados en el análisis de nutrientes, los cuales estimulan la formación de células epidérmicas, aumentando elongación foliar y la actividad de la H+-ATPasa a nivel de membranas plasmalema y tonoplasto $[28,29]$.

Con respecto al efecto bio estimulante en el incremento de número de hojas por la cepa nativa de Trichoderma sp., los resultados concuerdan con investigaciones efectuadas por diferentes autores [11,19] quienes reportan incrementos en número de hojas después de inocular cepas nativas de Trichoderma lignorum y T. harzianum a una concentración de $1 \times 10^{-8} \mathrm{UFC} / \mathrm{mL}$ en cultivos de caña de azúcar y maracuyá; aquello, posiblemente se debe a la capacidad que tiene el hongo para acidificar el microambiente alrededor de las raíces, liberando ácidos orgánicos, producir metabolitos quelantes que secuestran cationes y actividad redox; estos mecanismos vuelven solubles minerales y compuestos como oxido de manganeso, zinc $y$ fosfato de calcio que generalmante son poco solubles [30]; además la producción de enzimas hidrolíticas degradan constituyentes de la pared celular de microorganismos pudiendo ser utilizados como nutrientes, lo cual puede explicar en parte la capacidad de Trichoderma harzianum para aumentar la biomasa vegetal [31].

\section{Pigmentos clorofílicos}

En la Fig. 3 se muestran los efectos de las aplicaciones de Trichoderma sp. y lixiviados de vermicompost bovino (LVCB) sobre el contenido de clorofilas, demostrando que existen diferencias $(p<0,05)$ entre las medias de los tratamientos. El contenido de clorofila resultó en todos los tratamientos con Trichoderma y LVCB superiores al testigo. Los tratamientos que 
indujeron los mayores pigmentos clorofílicos fueron Trichoderma $6 \mathrm{~mL} 5$ y 15 DDS, LVCB1:10, $4 \mathrm{~mL}$ 5- DDS. Las dosis de Trichoderma lograron incrementar pigmentos clorofílicos entre $17 \%$ y $23 \%$, y el LVCB de igual manera $23 \%$ en relación a pigmentos clorofílicos de plantas sin aplicación.

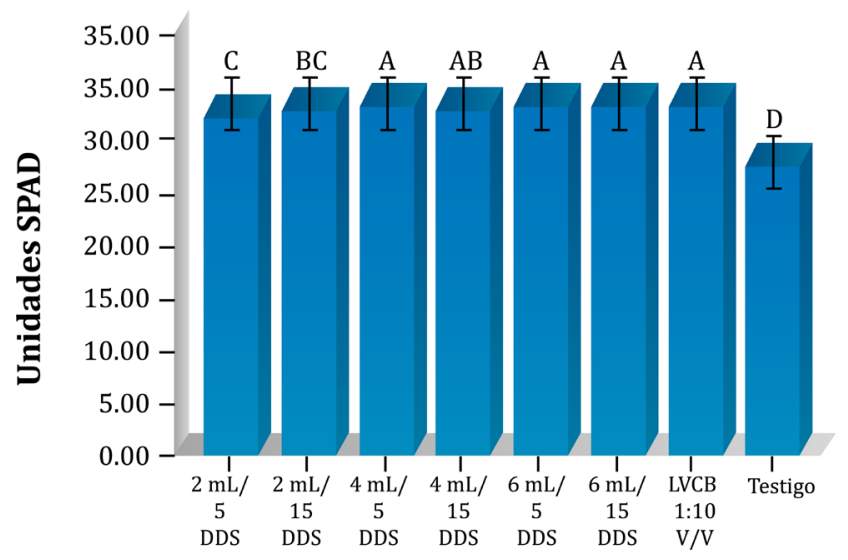

Fig. 3: Valores medios de pigmentos clorofílicos (unidades SPAD) a las aplicaciones de Trichoderma sp. y lixiviados de vermicompost bovino (LVCB). Medias con una letra común no son significativamente diferentes para el test de Duncan ( $\mathrm{p}>$ $0,05)$

Los resultados concuerdan con investigación de Jaimes y Moya $[32,33]$ efectuada en cultivo de chile y cebada que reportan incrementos de pigmentos clorofílicos después de aplicar cepas nativas de $T$. asperellum y Trichoderma spp. a concentraciones de $1 \times 10^{-8} \mathrm{UFC} / \mathrm{mL}$, mismas que se inocularon al momento del trasplante del chile y 24 horas antes de la siembra de la cebada.

Szczech M et al. [34] mencionan que la acumulación de clorofilas es favorecida debido a la aplicación de nitrógeno. En este contexto, aislados de Trichoderma sp. influyeron positivamente en la absorción de nitrógeno, generando un crecimiento significativo en cultivos de gramíneas [21] y en cultivo de col al aumentar la actividad enzimática del suelo incluida la ureasa, misma que es fundamental para trasformar urea en amonio proporcionando más nitrógeno al suelo [35]. En solanáceas, el índice de clorofila se incrementó significativamente con la aplicación de lixiviados de vermicompost de estiércol bovino y caprino [36] debido a que las sustancias húmicas permitieron el apilamiento mejor organizado y estructurado de los tilacoides aumentando el contenido de clorofilas en hojas de crisantemo acelerando la tasa de fotones para ser absorbidos, transferidos y convertidos en el cloroplasto [37]; además, los compuestos húmicos contenidos en el lixiviado aumentan el contenido de clorofila a través de la estimulación de la absorción de elementos nutricionales como el nitrógeno, que es necesario para la biosíntesis de clorofila y la actividad de la rubisco que resulta de la fotosínteis [38-40].

En general, la clorofila se relaciona con la concentración de nitrógeno y magnesio constituyendo estructuras de pigmentos fotosintéticos [41,42] y estos elementos nutricionales están presentes en el lixiviado de vermicompost, tal como lo demuestra la composición química; se ha sugerido que las características fisicoquímicas de los compuestos de ácidos húmicos del vermicompost pueden acelerar en plantas de achicoria la absorción de nitrógeno y hormonas reguladoras del crecimiento [43].

En la presente investigación no existieron factores intrínsecos que modifiquen el desenvolvimiento de las plántulas de arroz; en cuanto al requerimiento hídrico, el cultivo estuvo siempre con agua tal como lo indica la fitotecnia del cultivo, situación que pudo influenciar en el contenido de clorofilas, las cuales incrementan significativamente con el aumento de la humedad del suelo [44]. En un estudio sobre la aplicación de fuentes de nutrición orgánicas en el cultivo de arroz, se determinó que este tipo de nutrición aplicada sobre el cultivo aumentó notablemente el crecimiento vegetativo [10], de igual manera a lo ocurrido en la investigación efectuada en variedad SFL 11 en fase de semillero.

\section{CONCLUSIONES}

La aplicación de la cepa nativa de Trichoderma sp. incrementó el número de hojas, masa seca foliar y pigmentos clorofílicos en las plántulas de arroz variedad SFL-11.

La imbibición de la semilla de arroz variedad SFL 11 en el bioestimulante líquido de vermicompost de estiércol bovino en dilución 1:10 indujo incrementos significativos en masa seca foliar, número de hojas y el contenido de clorofilas en las posturas de arroz variedad SFL-11.

Se recomienda utilizar la cepa nativa de Trichoderma sp. en concentración de $1 \times 10^{-8}$ UFC/ $\mathrm{mL}$ en dosis de $4 \mathrm{~mL}$ aplicado a los 15 días después de la siembra y de $6 \mathrm{~mL}$ aplicado a los 5 y 15 días después de la siembra, y someter a imbibición la semilla de arroz en $10 \mathrm{~mL}$ de lixiviado de 
vermicompost de estiércol bovino por litro de agua durante 24 horas en pre-germinación.

\section{AGRADECIMIENTOS}

Por el significativo aporte para el desarrollo de esta investigación, hacemos extensivos nuestros sinceros agradecimientos al Ministerio de Agricultura y Ganadería- Dirección Distrital Manabí- Proyecto de Innovación Tecnológica Participativa y Productividad Agrícola (PITPPA), Asociación Artesanal La Pepa de Oro de Membrillo y Asociación Agrícola Paraíso de los Ceibos. Asi mismo, al productor de arroz Leonardo Delgado Delgado, socio de la Cooperativa de Producción Agrícola Leonidas Plaza Gutiérrez, al Dr. José Montesdeoca González por el soporte técnico a nivel de laboratorio al inicio del trabajo de investigación y a la analista de laboratorio Katty Ormaza Cedeño de la ESPAM-MFL.

\section{REFERENCIAS}

[1] Campoverde JF. Principales enfermedades que afectan el cultivo del arroz Oryza sativa L. en la zona de Arenillas provincia de El Oro (Bachelor's thesis, Machala: Universidad Técnica de Machala); 2016.

[2] Zambrano CE, Andrade MS, Rodríguez C, Villamil W. Factores que inciden en la productividad del cultivo de arroz en la provincia Los Ríos. Revista Universidad y Sociedad. 2019; 11 (5):270-277.

[3] Divya J, Belagali S. Effect of chemical fertilizers on physico-chemical characteristics of agricultural soil samples of Nanjangud taluk, Mysore District, Karnataka, India. The Ecoscan. 2012; 6 (3\&4):181187.

[4] Das A, Patel D, Munda G, Ramkrushna G, Kumar $\mathrm{M}$, Ngachan $\mathrm{S}$. Improving productivity, water and energy use efficiency in lowland rice (Oryza sativa) through appropriate establishment methods and nutrient management practices in the mid-altitude of north-east Experimental Agriculture. 2014; 50 (3):353-375.

[5] Shukla S, Meena R, Verma V, Singh RK. Effect of Various Sources of Nutrition on Plant Growth, Yield and Quality of Rice (Oryza sativa L.) cv MTU 7029. Environment and Ecology. 2017; 35 (2B):11681172.

[6] Dahiphale A, Giri D, Thakre G, Giri M. Effect of integrated nutrient management on yield and yield contributing parameters of scented rice. Annals of Plant Physiol. 2003;17(1):24-26.

[7] Du Jardin P. Plant biostimulants: definition, concept, main, categories and regulation. Scientia Horticulturae. 2015; 196:3-14.
[8] Canellas LP, Olivares FL, Aguiar NO, Jones DL, Nebbioso A, Mazzei P, Piccolo A. Humic and fulvic acids as biostimulants in horticulture. Scientia Horticulturae. 2015; 196:15-27.

[9] Calderín A, Guridi F, Louro R. Effects of Humic Materials on Plant Metabolism and Agricultural Productivity. Emerging Technologies and Management of Crop Stress Tolerance. Biological Techniques. 2014; 1: 449-466

[10] Núñez L, Pavone D. Tratamiento biológico del cultivo de arroz en condiciones de vivero empleando el hongo Trichoderma spp. 2014. Interciencia [Internet]. 2014; 39 (3): 3-39. Disponible en: https://www.interciencia.net/wpcontent/uploads/2017/10/185-c-PAVONE-7.pdf

[11] Cock L, García C, Hernández L. Biofertilización, una alternativa al uso de fertilizantes químicos en caña de azúcar (Saccharum officinarum). Alimentos Hoy. [Internet]. 2011; 20 (24):69-82. Disponible en: https://alimentoshoy.acta.org.co/ index.php/hoy/article/view/18

[12] Sotomayor A, González A, Kang J, Villavicencio A, Jackson T, Viera, W. 2019. Effect of the application of microorganisms on the nutrient absorption in avocado (Persea americana Mill.) seedlings. The Journal of the Korean Society of International Agriculture.2019; 31(1): 17-24.

[13] Harman GE, Petzoldt R, Comis A, Chen J. Interactions between Trichoderma harzianum strain T22 and maize inbred line Mo17 and effects of these interactions on diseases caused by Pythium ultimum and Colletotrichum graminicola. Phytopathology. 2004; 94 (2):147-153.

[14] Suquilanda M. Manejoagroecológico deplagas. Ministerio de Agricultura, Ganaderia, Acuacultura y Pesca Ecuador. [Internet].2017:28-30. Disponible en: https://docplayer.es/54127061-Manejoagroecologico-de-plagas-manuel-b-suquilandavaldivieso.html

[15] Zhao L, Zhang Y. Effects of phosphate solubilization and phytohormone production of Trichoderma asperellum Q1 on promoting cucumber growth under salt stress. Journal of Integrative Agriculture. 2015; 14 (8): 1588-1597

[16] Martínez B, Infante D, Reyes Y. Trichoderma spp. y su función en el control de plagas en los cultivos. Revista de Protección Vegetal. 2013; 28 (1):1-11

[17] González MA. Evaluación agronómica de dos variedades de arroz Oryza sativa L. con fertilización nitrogenada y dosis de mejoradores orgánicos (Bachelor's thesis, Guayaquil: Universidad de Guayaquil); 2016.

[18] Torres A, Ardisana E, Del Valle G, Cue J, Téllez 0. Efectos del BIOSTAN® en los índices de crecimiento y los pigmentos fotosintéticos de 
Phaseolus vulgaris L. La Técnica ISSN 2477-8982. 2017;(18):25-35

[19] Cubillos-Hinojosa J, Valero N, Mejía L. Trichoderma harzianum como promotor del crecimiento vegetal del maracuyá (Passiflora edulis var. flavicarpa Degener). Agron colomb [Internet] 2009; 27 (1):81-86. Disponible en: https://revistas.unal.edu.co/index.php/agrocol/ article/view/11363/37730

[20] Camargo-Cepeda DF, Ávila ER. Efectos de Trichoderma sp. sobre el crecimiento y desarrollo de la arveja (Pisum sativum L.). Ciencia y Agricultura. 2014; 11 (1):91-100.

[21] Vinale F, Sivasithamparam K, Ghisalberti EL, Marra R, Woo S, Lorito M. Trichodermaplant-pathogen interactions. Soil Biology and Biochemistry. 2008; 40 (1):1-10.

[22] López J, Pelagio R, Herrera A. Trichoderma as biostimulant: exploiting the multilevel properties of a plant beneficial fungus. Scientia Horticulturae. 2015; 196:109-123

[23] Casa MG. Evaluación del efecto de los ácidos húmicos sobre los parámetros de calidad en plántulas de tomate (Solanum lycopercicum L.) [Internet] Universidad Técnica de Cotopaxi; 2010. Disponible en: http://repositorio.utc.edu.ec/ bitstream/27000/758/1/T-UTC-0586.pdf

[24] Ochoa J, German J. Efecto del uso de ácidos húmicos, fúlvicos y su interacción con fertilizante nitrogenado en el crecimiento de plántulas de café (Coffea arabica L.) en vivero [Internet]. Honduras: Escuela Agrícola Panamericana Zamorano; 2017. Disponible en: https://bdigital.zamorano.edu/ bitstream/11036/6012/1/CPA-2017-075.pdf

[25] Hernández R, García A, Portuondo L, Muñoz S, Berbara R, Izquierdo F. Protección antioxidativa de los ácidos húmicos extraídos de vermicompost en arroz (Oryza sativa L.) var. IACuba30. Revista de Protección Vegetal. 2012; 27 (2):102-110

[26] Rodríguez J, Pérez J, Rodríguez J. Efecto de un bioestimulante natural sobre algunos parámetros de calidad en plántulas de tomate (Solanum Lycopersicum, L.) bajo condiciones de salinidad. Biotecnia. 2016; 18 (2): 11-15.

[27] Olaetxea M, De Hita D, Garcia A, Fuentes M, Baigorri R, Mora V, Urrutia O, Erro J, Zamarreño M, Berbara R, Garcia J. Hypothetical framework integrating the main mechanisms involved in the promoting action of rhizospheric humic substances on plant root- and shoot- growth. Applied Soil Ecology. 2018; 123: 521-537

[28] Canellas P, Olivares F, Okorokova A, Facanha A. Humic acids isolated from earthworm compost enhance root elongation, lateral root emergence, and plasma $\mathrm{H}+-\mathrm{ATPa}$ activity in maize roots. Plant Physiology. 2002; 130: 1951-1957
[29] Zandonadi D, Canellas P, Façanha A. Indolacetic and humic acids induce lateral root development through a concerted plasmalemma and tonoplast $\mathrm{H}+$ pumps activation. Planta. 2006; 225(6): 1583-1595

[30] Altomare C, Norvell W, Björkman T, Harman G. Solubilization of phosphates and micronutrients by the plant-growth-promoting and biocontrol fungus Trichoderma harzianum Rifai 1295-22. Appl Environ Microbiol. 1999; 65 (7):2926-2933

[31] Rifai M. A revision of the genus Trichoderma. Mycological papers. 1969; 116: 1-56.

[32] Jaimes Cedillo JR. Microencapsulacion de (Bacillus subtilis y Trichoderma asperellum) y su efecto antagónico sobre (Fusarium oxysporum y Rhizoctonia solani) y en el desarrollo de plantas de chile (Capsicum annuum) [Internet] Buenavista: Repositorio Digital Universidad Autónoma Agraria Antonio Narro 2011. Disponible en: http: / / repositorio.uaaan.mx:8080/xmlui / handle/123456789/4316

[33] Moya P. Antagonismo y efecto biocontrolador de Trichoderma spp. sobre Drechslera teres, agente causal de la" mancha en red" de la cebada (Hordeum vulgare L. var. vulgare) [Internet]. Argentina: Sistema Nacional de Repositorios Digitales.; 2016. Disponible en: https://repositoriosdigitales. mincyt.gob.ar/vufind/Record/

[34] Szczech M, Nawrocka J, Felczyński K, Małolepsza U, Sobolewski J, Kowalska B, Maciorowski R, Jas K, Kancelista A. Trichoderma atroviride TRS25 isolate reduces downy mildew and induces systemic defence responses in cucumber in field conditions. Scientia Horticulturae. 2017; 224:17-26.

[35] Ji S, Liu Z, Liu B, Wang Y, Wang J. The effect of Trichoderma biofertilizer on the quality of flowering Chinese cabbage and the soil environment. Scientia Horticulturae. 2020; 262: 109069

[36] Torres A, Ardisana E, Téllez 0, Cue J, Muñoz J, Aguilar R, Bravo S. Respuesta del pimiento (Capsicum annuum L.) ante aplicaciones foliares de diferentes dosis y fuentes de lixiviados de vermicompost. Bioagro. 2019; 31 (3):213-220

[37] Fan H, Wang X, Sun, X, Li Y., Sun X, Zheng C. Effects of humic acid derived from sediments on growth, photosynthesis and chloroplast ultrastructure in chrysanthemum. Scientia Horticulturae. 2014; 177: 118-123.

[38] Sánchez E, Ruiz JM, Romero L, PreciadoRangel P, Flores-Córdova MA, Márquez-Quiroz C. ¿Son los pigmentos fotosintéticos buenos indicadores de la relación del nitrógeno, fósforo y potasio en frijol ejotero? Ecosistemas y recursos agropecuarios. 2018; 5 (15):387-398. 
[39] Delfine R, Tognetti E, Desiderio A. Effect of foliar application of $\mathrm{N}$ and humic acids on growth and yield of durum wheat, Agron. Sustain. Dev. 2005; (2): 183-191

[40] Khayyat E, Tafazoli S, Eshghi S. Effect of nitrogen, boron, potassium and zinc sprays on yield and fruit quality of date palm, American Eurasian J. Agric \& Environ Sci. 2007; 2(3): 289-296

[41] Mathivanan S, Kalaikandhan R, Chidambaram A, Sundramoorthy P. Effect of vermicompost on the growth and nutrient status in groundnut (Arachis hypogaea L.) Asian J Plant Sci Res.. 2013; 3 (2):1522.

[42] Zhang X, Huang G, Bian X, Zhao Q. Effects of root interaction and nitrogen fertilization on the chlorophyll content, root activity, photosynthetic characteristics of intercropped soybean and microbial quantity in the rhizosphere. Plant Soil Environ. 2013; 59 (2):80-88.

[43] Gholami H, Ghani A, Raouf F, Saharkhiz M, Hazrati $\mathrm{H}$. Changes in photosynthetic pigments and uptake of some soil elements by chicory supplied with organic fertilizers. Acta Ecologica Sinica. 2019; 39 (3):250-256.

[44] Torres A, López A, Hernández G, Díaz B. Influencia de la humedad del suelo y la fertilización sobre el contenido de pigmentos verdes en posturas de Citrus. Instituto Nacional de Ciencias Agrícolas de Cuba. Revista Cultivos Tropicales. 1984; 6 (4): 755-788. 